\title{
IEM-based Tone Injection for Peak-to-Average Power Ratio Reduction of Multi-carrier Modulation
}

\author{
Yang Zhang, Xiangmo Zhao, Jun Hou* and Yisheng An \\ School of Information Engineering, Chang'an University, \\ Xi'an, Shaanxi, 710064, P.R. China \\ [e-mail: jhou@chd.edu.cn] \\ *Corresponding author: Jun Hou
}

Received May 28, 2018; revised February 9, 2019; accepted April 3, 2019;

published September 30, 2019

\begin{abstract}
Tone Injection (TI) scheme significantly reduces the peak-to-average power ratio (PAPR) of Multicarrier Modulation (MCM). However, the computational complexity of the TI scheme rises exponentially with the extra freedom constellation number. Therefore, a novel immune evolutionary mechanism-based TI scheme is proposed in this paper to reduce the computational complexity. By restraining undesirable degeneracy during the processing, this IEM scheme can dramatically increase the population fitness. Monte Carlo results show that proposed IEM-based TI scheme can achieve a significant PAPR and BER improvement with a low complexity.
\end{abstract}

Keywords: wireless communication, multi-carrier modulation, peak-to-average power ratio, immune evolutionary mechanism 


\section{Introduction}

Multicarrier modulation (especially orthogonal frequency division multiplexing) has widely used in a number of standard systems, including the 3GPP LTE, IEEE $802.11 \mathrm{a} / \mathrm{g} / \mathrm{n}$ standard, and WiMAX standard, due to the merits of robustness to the selective fading and high spectral efficiency, etc [1]-[3].

However, the inherent high peak-to-average power ratio (PAPR) of MCM systems causes large signal distortion and inefficiency of the power amplifier (due to large back-off). Numerous PAPR reduction methods are thus obtainable in the references (see [4]-[8] and the references therein), among these solutions, tone injection (TI) [2], [9]-[15] is a distortion-less scheme that can reduce the PAPR dramatically without side information transmission or extra data rate loss. It uses hexagonal constellation [9] or extended constellations [10] to achieve a lower PAPR. However, the implementation requires solving an integer-programming problem, which belongs to a NP-hard problem. Therefore, one has to produce a good suboptimal solution, such as clipping projection [11], [15], cross-entropy [12], tabu search [13] and linear programming [14].

Inspired by quantum-inspired evolutionary algorithm [16] successfully utilized to resolve combinatorial optimization problem, a basic immune evolutionary algorithm is introduced in [17]. However, since degeneracy is not completely eliminated, it can be improved further. Consequently, in order to achieve better system performance, this paper introduces immune mechanism [18], [19] to the quantum computing. To preserve evolutionary mechanism's advantages, a novel immune evolutionary mechanism (IEM) is utilized for restraining the degeneracy during evolution, which improves the algorithmic efficiency and reduces the complexity. Thus, new IEM TI schemes are developed for PAPR reduction. Furthermore, in this paper, we use a specially hexagonal constellation to eliminate power increase caused by conventional tone injection. Monte Carlo results confirm the IEM-TI achieves significant improvement in BER and PAPR with a low complexity.

The outline of the rest in this paper is organized as follows. The MCM Model and problem formulation are described in Section II. Section III summarizes the concepts and principles of quantum computing and immune mechanisms. By introducing immune mechanism to the quantum computing, Section IV puts forth the proposed schemes to search the extra freedom constellation for TI. Section V presents the performance evaluation. Conclusion is summarized in Section VI.

\section{MCM Model and Problem Formulation}

\subsection{Model and PAPR Definition}

For a $N$ subcarriers MCM system with quadrature amplitude modulation (QAM), the outputs of the inverse discrete Fourier transform (IDFT) of signal $X_{k}$ is given by 


$$
X_{n}=\frac{1}{\sqrt{L N}} \sum_{k=0}^{L N-1} X_{k} \exp \left(\frac{j \cdot 2 \pi k n}{L N}\right), \quad n=0,1, \ldots, L N-1
$$

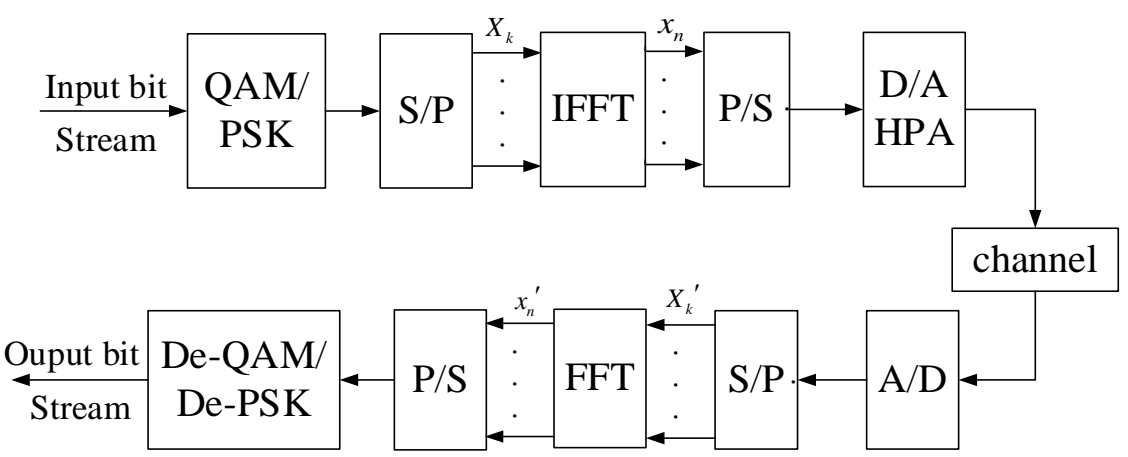

Fig. 1. Diagram of a traditional MCM system.

The PAPR definition in transmitted MCM signal can be expressed as

$$
P A P R=10 \cdot \log _{10} \frac{\max _{0 \leq n<N L-1}\left\{\left|x_{n}\right|^{2}\right\}}{E\left[\left|x_{n}\right|^{2}\right]}(d B) .
$$

Here, the $E[$.] denotes the expectation operation (Note that during the simulations, it is will be replaced by its mean value as a further simplification). Furthermore, as shown in [20], in order to keep a good approximation to the continuous signals, the MCM signal must oversampled by a factor that larger than 4.

\subsection{Tone Injection for PAPR Reduction}

Mapping the original MCM constellation to several equivalent constellations, tone injection [2] can use any of the equivalent constellation to carry the same information. Mathematically, for an $M$-ary square QAM signal, the symbols after TI scheme can be expressed as [2]

$$
x_{n}^{\prime}=\frac{1}{\sqrt{n}} \sum_{k=0}^{N-1}\left(X_{k}+p_{k} \cdot \Delta+j q_{k} \cdot \Delta\right) \exp \left(\frac{j 2 \pi n}{L N}\right)
$$

where $\sqrt{M}$ represent the level per dimension, $p_{k}$ and $q_{k}$ are integers, and $d$ is the minimum distance of the constellation points. In addition, literature [3] shows that in order to keep the BER performance at the receiver maintain the same level, $\Delta$ must be larger than $d \sqrt{M}$.

On one hand, taking the complexity problem into account, assume only one candidate constellation for each MCM symbol can be chosen to shift, then, the optimization problem in TI scheme can be given by

$$
\begin{aligned}
& \min f(b)=\left|x_{n}^{\prime}(b)\right|^{2} \\
& \text { subject to }: b \in\{0,1\}^{N} .
\end{aligned}
$$


Equation (4) has been proven to be a non determined-polynomial-time complete problem. Therefore, an efficient IEM-TI scheduling scheme will be proposed in next section.

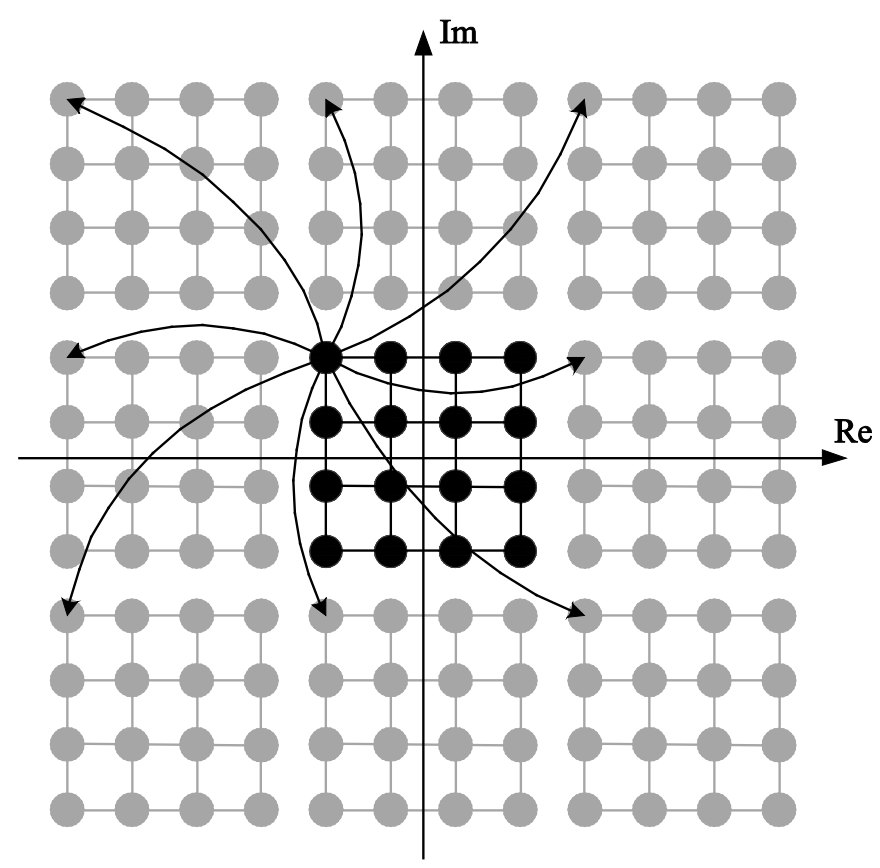

Fig. 2. Constellation expansion with eight replications for a MCM symbol.

On the other hand, considering that hexagonal lattice [21] is the most dense packing in two dimensions. Therefore, in order to reduce the PAPR without increasing the signal power, a hexagonal constellation (HC) is introduced to TI [9]. In this case, for each constellation point, the decision region is $\sqrt{3} d^{2} / 2$, and the new transmit signal can then be expressed as

$$
x_{n H C}^{\prime}=x_{n H C}-\frac{1}{\sqrt{N}} \sum_{k=0}^{N-1} b_{k} X_{k H C} e^{j 2 \pi n k / L N},
$$

where $x_{n H C}$ is the same as $x_{n}$ in Eq. (1) and $b_{k}$ is defined in Eq. (4). In addition, the $X_{k H C}=X_{k}+p_{k} \cdot \Delta+j q_{k} \cdot \Delta$.

\subsection{Cubic Metric in MCM Systems}

It has been shown in [22] that for a real power amplifier (PA) in a cellular handset, the cubic metric (CM) can predict the power capability reduction effectively. Since its accuracy in many devices and signals, 3GPP members chosen CM as a method to determine PA power derating. This method has shown [23] a better performance than the PAPR to predict derating for the WCDMA and LTE signals. The CM in a typical MCM system is defined as

$$
C M=\frac{R C M-R C M_{r e f}}{K},
$$


note that the $R C M$ denote the raw cubic metric. The $R C M$ for a given signal $d(t)$ can be defined as follows.

$$
R C M=20 \log \left[r m s\left[\left(\frac{|d(t)|}{r m s[d(t)]}\right)^{3}\right]\right],
$$

where $\operatorname{rms}[d(t)]$ is the root-mean-square of a given signal $d(t)$.

Here, the parameter $K$ and $R C M_{\text {ref }}$, shown in [23], are introduced to complete estimate of the power derating required to meet a certain adjacent channel leakage ratio (ACLR). For example, the values of parameter $R C M_{\text {ref }}$ and $K$ in LTE downlink are1.52dB and 1.56, respectively. Based on this, in this paper, the $C M$ performance of the proposed IEM scheme are also tested.

\section{Concepts of Evolutionary and Immune Mechanisms}

In order to puts forth the proposed scheme for PAPR reduction, in this Section we will first introduce the concepts and principles of quantum computing and immune mechanisms.

\subsection{Basics of Quantum Computing}

In conventional computing, one bit is either in the state-' 1 ' or state-' 0 '. The quantum analogue is a Quantum bit or a $Q$-bit. Note that a $Q$-bit is probably in the state-' 1 ' or state-' 0 ', or in any superposition of these two states. The state $|\Psi\rangle$ can be defined as

$$
|\Psi\rangle=\alpha|0\rangle+\beta|1\rangle, \text { or }|\Psi\rangle=\left[\begin{array}{l}
\alpha \\
\beta
\end{array}\right],
$$

where $\alpha$ and $\beta$ are the probability of the $Q$-bit in the corresponding state-' 1 'or state-' 0 ', and $|\alpha|^{2}+|\beta|^{2}=1$. In other words, a $Q$-bit defines an angle $\theta$, where $\cos (\theta)=|\alpha|$ and $\sin (\theta)=|\beta|$. A $Q$-bit individual is denoted as a string of $V$ in $Q$-bits [14]

$$
|\Psi\rangle=\left[\begin{array}{c|c|c|c}
\alpha_{1} & \alpha_{2} & \cdots & \alpha_{V} \\
\beta_{1} & \beta_{2} & \cdots & \beta_{V}
\end{array}\right],
$$

where $\left|\alpha_{i}\right|^{2}+\left|\beta_{i}\right|^{2}=1, i=1,2, \ldots V$. Moreover, quantum gate (denote as the $Q$-gate) [16], [18] is proposed to update the $Q$-bit individuals of the probabilities of $\alpha$ and $\beta$. Here, note that this $Q$-gate is a reversible gate and can be denoted as a unitary operator $Z$ acting on the $Q$-bit basis states satisfying $Z^{\dagger} Z=Z Z^{\dagger}$, where $Z^{\dagger}$ is the hermitian adjoint of $Z$. A system with $s Q$-bits can represent $2^{s}$ states at the same time. By updating these states with a certain $Q$-gate, the best solution is obtained.

\subsection{Immune Mechanism}

Immune concepts and methods in an evolutionary process aim to find the optimal solution 
by using the locally characteristic information. Local information can be utilized to restrain useless and reduplicated work and involve in the global searching, in order to remove the blind mutation. The immune algorithm, like the immune phenomenon in nature, refrains degeneracy in evolutionary process, which, in turn, making the population fitness rising steadily [19]. The immunity operation can be achieved by two steps [24], [25]: 1) vaccination; 2) Immune selection. The former can raise the average fitness of population and the latter can prevent the useless and repetitive work, e.g., deterioration. These two steps can be described as follows.

- Vaccination

Assume the individual $x$ is a potential solution (bit sequence) for an integer optimization problem. Vaccination of $x$ means to modify some bits of $x$ according to the priori knowledge to increase the probability of increased fitness. In the next Section, we will give the general steps to realize it.

- Immune Selection

First, if the vaccination individual [19] has worse fitness value than the original one (parent), then the parent not the vaccination individual will participate the next competition. The current and parent populations are sorted based on individual fitness and the contribution to the diversity of the population. The individuals whose fitness value are better than the average and differ greatly from the best individual will survive with a larger probability. Note that this step not only guarantees the effective transmission of evolutionary information but also keeps the diversity of population.

In Section 4, the vaccination schedule and the selection method will compared with other methods by simulations.

\section{The Proposed Algorithm and Its Complexity}

By introducing immune mechanism to the quantum computing, in this section, we first give the general Immune Evolutionary Mechanism (IEM) steps, and then put the IEM to the TI method for PAPR reduction.

\subsection{Immune Evolutionary Mechanism}

For general integer optimization problem using the IEM, in every generation, both the fitness and bit values of the solutions are compared. For example, if one solution $b_{i}$ is better than another one $b_{j}$ and if the bit values differ, then the $Q$-gate operator is used to update the corresponding bits. Therefore, the worse solution $b_{j}$ moves slightly toward to the better one $b_{i}$. In addition, to prevent this operation being irreversible, elitism is introduced [18]. The elitist at generation $t+1$ is achieved by the optimal solution in the $t$ generation. Since the elitist is compared with every individual, the information collected during search evolution is not only maintained at the individual level but also periodically communicated to the entire population.

Similar to other probabilistic evolutionary methods, at generation $t$, IEM retains $Q$-bit individuals at the size of pop, e.g., $Q(t)=\left\{q_{1}^{t}, q_{2}^{t}, \ldots, q_{p o p}^{t}\right\}$, where $q_{i}^{t}$ is the $Q$-bit individual 


$$
q_{i}^{t}=\left[\begin{array}{c|c|c|c}
\alpha_{i 1}^{t} & \alpha_{i 1}^{t} & \ldots & \alpha_{i V}^{t} \\
\beta_{i 1}^{t} & \beta_{i 1}^{t} & \ldots & \beta_{i V}^{t}
\end{array}\right] .
$$

Here, the length of the Q-bit individual is defined as $V$, where $i=1,2, \ldots, V$. In principle, the IEM method is iteratively running in three steps:

Step 1. By observing the $Q$-gate to generate the random binary solutions in $P(t)$, where $B(t)=\left\{b_{1}^{t}, b_{2}^{t}, \ldots, b_{\text {pop }}^{t}\right\}$ and $b_{i}^{t}=\left\{b_{i 1}^{t}, b_{i 2}^{t}, \ldots, b_{i V}^{t}\right\}$ at generation $t$. In the PAPR problem, the binary value $b_{i k}^{t}$ is determined by

$$
b_{i k}^{t}=\left\{\begin{array}{l}
-1, \quad \text { if }\left|\alpha_{i k}^{t}\right|^{2}>\operatorname{random}[0,1) \\
1, \quad \text { if }\left|\alpha_{i k}^{t}\right|^{2}<\operatorname{random}[0,1)
\end{array} .\right.
$$

Step 2. Performing immuning on $B(t)$ to increase the fitness of population.

Vaccination: it can be achieved by the following steps: 1) Revise the phase information of the selected individual according to the vaccination schedule; 2) Remove the overlapping individuals in the past or current population(for $b_{j}^{t}$, if $b_{j}^{t}=b_{i}^{t}$ or $b_{j}^{t}=b_{i}^{t-n}$, which means $b_{j}^{t}$ already chosen in the population), then regenerate a new individual. After that, calculate the fitness value of the corresponding individual.

To modify the IEM-based TI candidate we need follow these steps to implement: based on all fitness value list the population; Then, pick the individual phase information with great difference to the best one (maximum $r$ bits). By using the following equation, it can be quickly achieve the corresponding fitness value for the purpose of the complexity reduction,

$$
f_{\text {modified }}=f_{\text {original }}-2 b_{i r} \cdot x^{r}
$$

By using Eq. (12), different individuals can be obtained.

Immune selection: this step can be achieved by the following procedures: sort the current and parent population based on individual fitness and the contribution to the diversity of the population, so that the fitness value better than the average and greatly differ from the best individual can survive.

Step 3. Modifying the probabilities $\alpha$ and $\beta$ by using $Q$-gate to update the $Q$-bit individuals. The $Q$-gate can be expressed as

$$
\left[\begin{array}{c}
\alpha_{i j}^{t+1} \\
\beta_{i j}^{t+1}
\end{array}\right]=\left[\begin{array}{cc}
\cos \left(\Delta \theta_{i j}^{t}\right) & -\sin \left(\Delta \theta_{i j}^{t}\right) \\
\sin \left(\Delta \theta_{i j}^{t}\right) & \cos \left(\Delta \theta_{i j}^{t}\right)
\end{array}\right] \cdot\left[\begin{array}{c}
\alpha_{i j}^{t} \\
\beta_{i j}^{t}
\end{array}\right],
$$

where $\Delta \theta$ is the rotation angle parameter of each $Q$-bit. Fig. 1 shows the rotation gate of individuals. Note that $\Delta \theta$ can control the convergence speed, large $\Delta \theta$ may converge to a local optimum solutions. Ref. [16] give a details design method of $\Delta \theta$. 


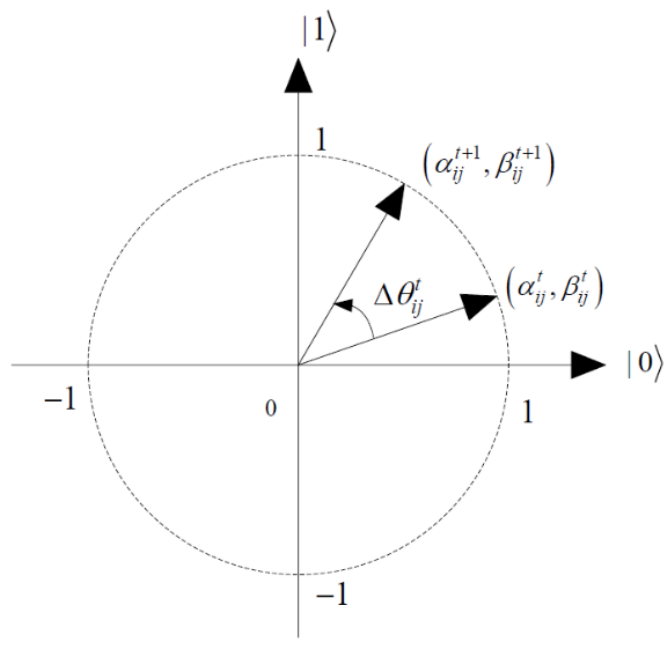

Fig. 3. Rotation gate for Q-bit individuals.

\subsection{Structure of the Proposed IEM}

The structure of IEM can now be summarized as follows:

\section{Procedure IEM}

$$
t \leftarrow 0
$$

1. initialize $Q(t)$, all qubits are set to $1 / \sqrt{2}$

2. make $B(t)$ by observing the states of $Q(t)$ from Eqs. (10) and (11)

3. evaluate $B(t)$ and store the best solutions among $B(t)$ into $B_{\text {best }}(t)$

while (not termination-condition) do

$t \leftarrow t+1$

4. make $B(t)$ by observing the states of $Q(t-1)$ and Eq. (11)

5. perform Immune (Step 2 and Eq.(12)) on $B(t)$

6. evaluate $B(t)$ by using Eq. (14)

7. update $Q(t)$ using Q-gates:(see Eq. (13) and APPENDIX) learn_model $\left(B_{i}^{t}, B_{\text {best }, i}^{t}, Q_{i}^{t}\right)$

8. store the best solutions among $B(t-1)$ and $B(t)$ into $B_{\text {best }}(t)$

9. store the best solution $\boldsymbol{b}$ among $B_{\text {best }}(t)$

10. if (migration-condition) then

migrate $\boldsymbol{b}$ or $\boldsymbol{b}_{i}^{t}$ to $B(t)$ globally or locally, respectively

end if

end while

A description of the learning model in detail are given in the Appendix. In the procedure, the best solutions in generation $t-1$ among $B_{\text {best }}(t-1)$ and $t$ among $B(t)$ are chosen and compared 
to the best in $B_{\text {best }}(t)$, the better one will be stored into new best solution $b$. Note that the algorithm running in the while loop until meet the termination condition.

Each solution must set a fitness function. Clearly, the candidate constellation in (5) maintain the average power unchanged. Therefore, the fitness function of the IEM-TI MCM may be expressed as

$$
\text { fitness }\left(b_{i}\right)=\min _{0 \leq n \leq N L-1}\left|x^{\prime}\left(b_{i}\right)\right|^{2} \text {. }
$$

Moreover, in IEM-TI, the not termination-condition is that $\operatorname{PAPR}\left(x^{\prime}\left(b_{i}\right)\right)$ larger than the predetermined threshold and the migration-condition is $\operatorname{PAPR}\left(x^{\prime}\left(b_{i}\right)\right)$ meets the requirement or maximum iteration number is achieved.

\subsection{Analysis of the Computational Complexity}

The sample number determines the total computational complexity when a population based PAPR searching method (see [10], [11], and [13]) is used. If the population size and generation value and are pop and Gen, then, its complexity can be shown as the number of samples, e.g., $p o p \times G e n$ (Here, each sample is calculated with a $O(N \log N)$ multiplications in fast Fourier transform). Note that this complexity analysis covers any population search-based PAPR reduction schemes.

In addition, for a given $f_{\text {original }}, N$ additions is needed in Equation (11) to obtain $f_{\text {modified. }}$. Therefore, compared with other methods, IEM increases the addition complexity while maintain the multiplication complexity constant. Next Section will show the performance evaluation of these schemes by simulations.

\section{Performance Evaluation}

In this section, the performance of the IEM-TI algorithm is evaluated by simulating $10^{5}$ MCM symbols under quadrature amplitude modulation with a 256 subcarriers. The system simulation parameters are given in Table 1.

Table 1. Simulation Environment

\begin{tabular}{|c|c|}
\hline System Parameter & Simulation Environment \\
\hline \hline Modulation Scheme & 64-QAM \\
\hline subcarriers number & 256 subcarriers \\
\hline SSPA & $6.5 \mathrm{~dB}$ saturation point, $p=3$ \\
\hline Oversampling factor & 4 \\
\hline$Q$-bit angle & $\Delta \theta=0.01 \pi$ \\
\hline
\end{tabular}

Furthermore, three population-based schemes, cross-entropy (CE-TI) [12], Parallel TS-TI [13], and linear programming(LP-TI) [14] are chosen for comparison. "IEM1" and "IEM2" in the figures are given in Appendix. 


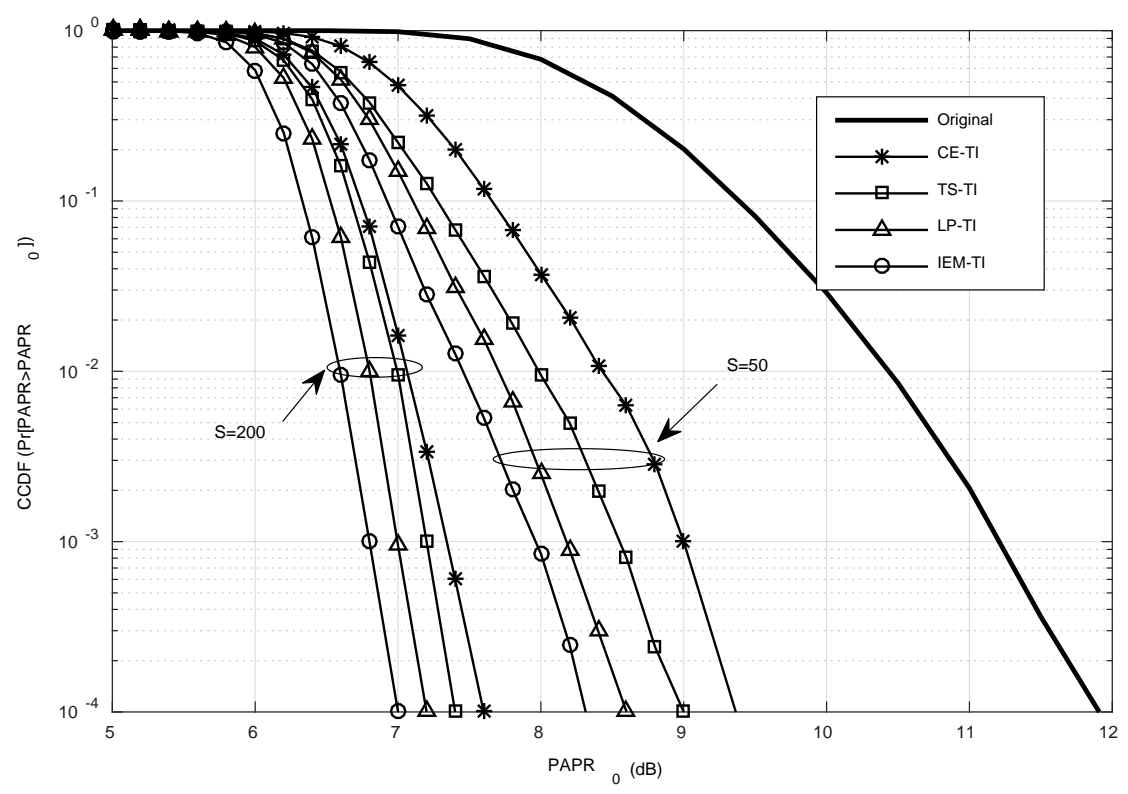

Fig. 4. PAPR for various TI techniques in various scenarios.

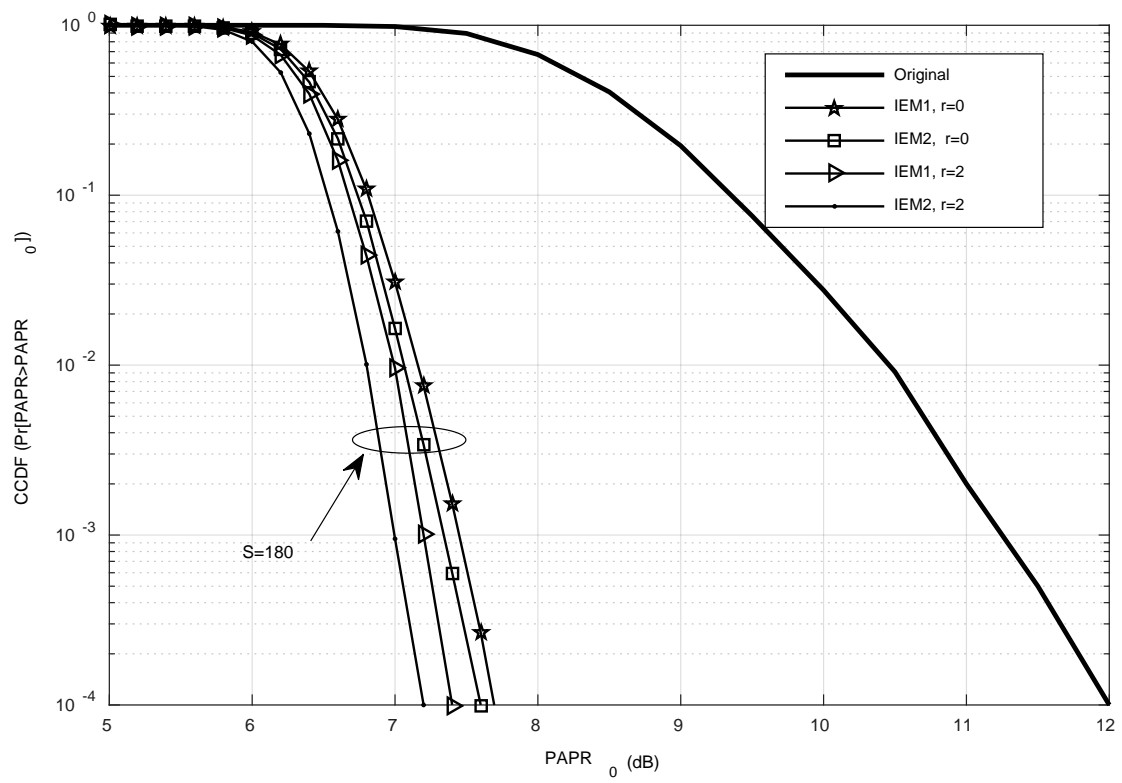

Fig. 5. CCDF of PAPR with $S=180$ of the IEM1and IEM2.

Figs. 4 and 5 depicts the PAPR reduction of various schemes.(Here, the parameter of the parallel TS in [13] as described below. The number in parallel is pop $=4$ and the move $=11$. Hence, the search number of parallel TS is pop multiply move. In addition, the crossover search number is given by $\mathrm{c}=C_{4}^{2}=6$. Similarly, we can obtain the search number under one iteration of the parallel TS is pop $\times$ move $+c=50$ ) As shown, the proposed IEM-TI schemes 
outperform the other schemes with the same computational complexity. Specially, to obtain a fixed PAPR level at generation value $S=50$, the proposed IEM scheme shows better performance than the CE-TI, Parallel TS-TI and the LP-TI schemes. When a sixty population size and the three generation value are used, the IEM1 and IEM2 schemes with $r=2$ gains about $0.5 \mathrm{~dB}$, as compared to IEM1 and IEM2 with $r=0$, respectively. Complexity comparisons are shown in Table 2.

Table 2. Complexity at $10^{-3}$ PAPR

\begin{tabular}{|c|c|c|}
\hline Algorithm & Number of Samples & PAPR \\
\hline CE-TI & 150 & $8.07 \mathrm{~dB}$ \\
\hline Parallel & {$[(11 \times 4)+6] \times 3=150$} & $7.91 \mathrm{~dB}$ \\
\hline LP-TI & 150 & $7.58 \mathrm{~dB}$ \\
\hline IEM-TI, $r=0$ & $50 \times 3=150$ & $7.05 \mathrm{~dB}$ \\
\hline
\end{tabular}

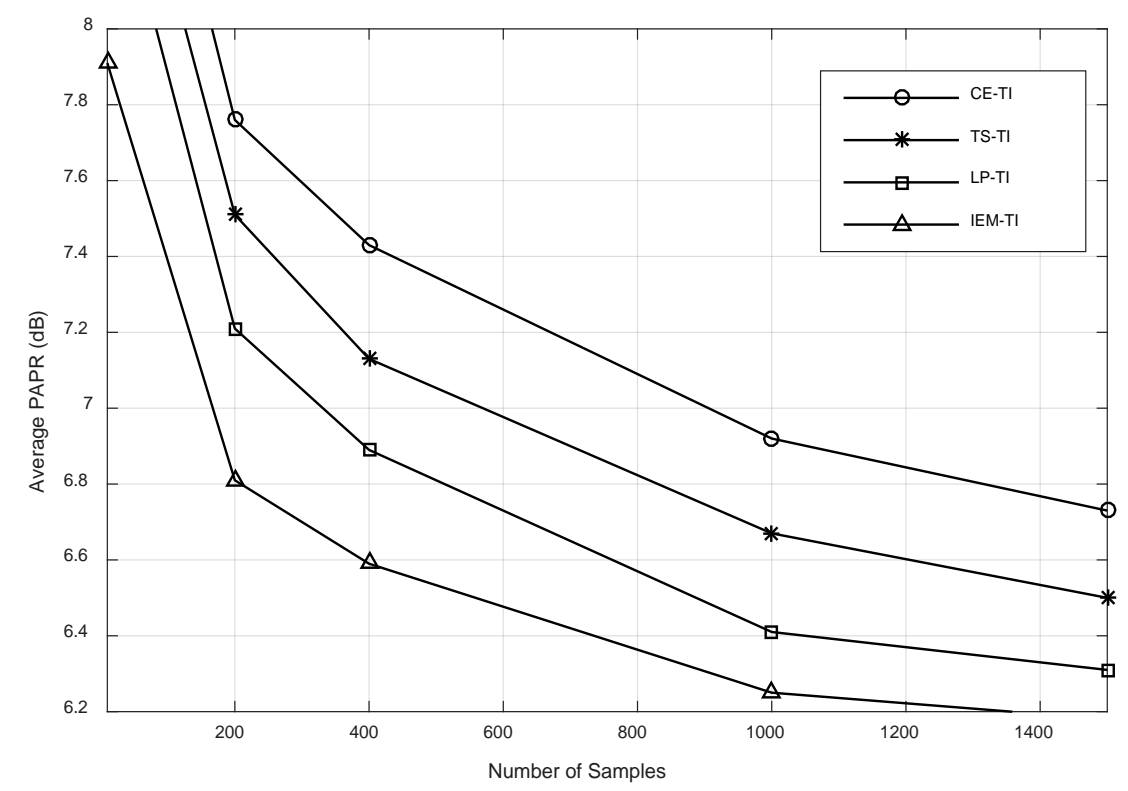

Fig. 6. PAPR for CCDF at $10^{-3}$ vs computational complexity for various schemes.

Fig. 6 compares the CCDF of PAPR with CE-TI, Parallel TS-TI, LP-TI and IEM schemes for the same complexity. The IEM schemes achieve much smaller PAPR than other schemes. Although the PAPR performance is improved with increase of the samples, the PAPR of IEM-TI with 400 samples is only less $0.3 \mathrm{~dB}$ than that of 1200 samples. Therefore, 400 samples can be a suitable choice for our proposed IEM schemes. Fig. 7 shows the comparison of cubic metric. As seen, the IEM-based TI scheme also plays a good CM performance during the simulations. 


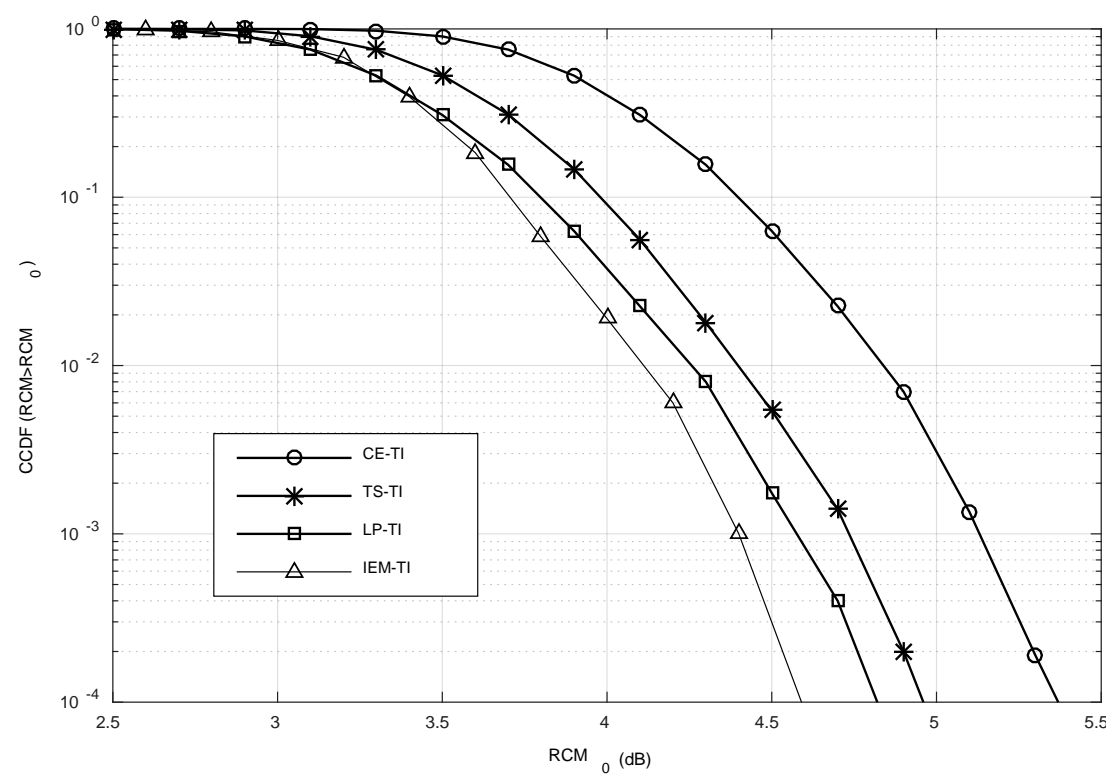

Fig. 7. CCDFs of the raw cubic metric comparison for various schemes.

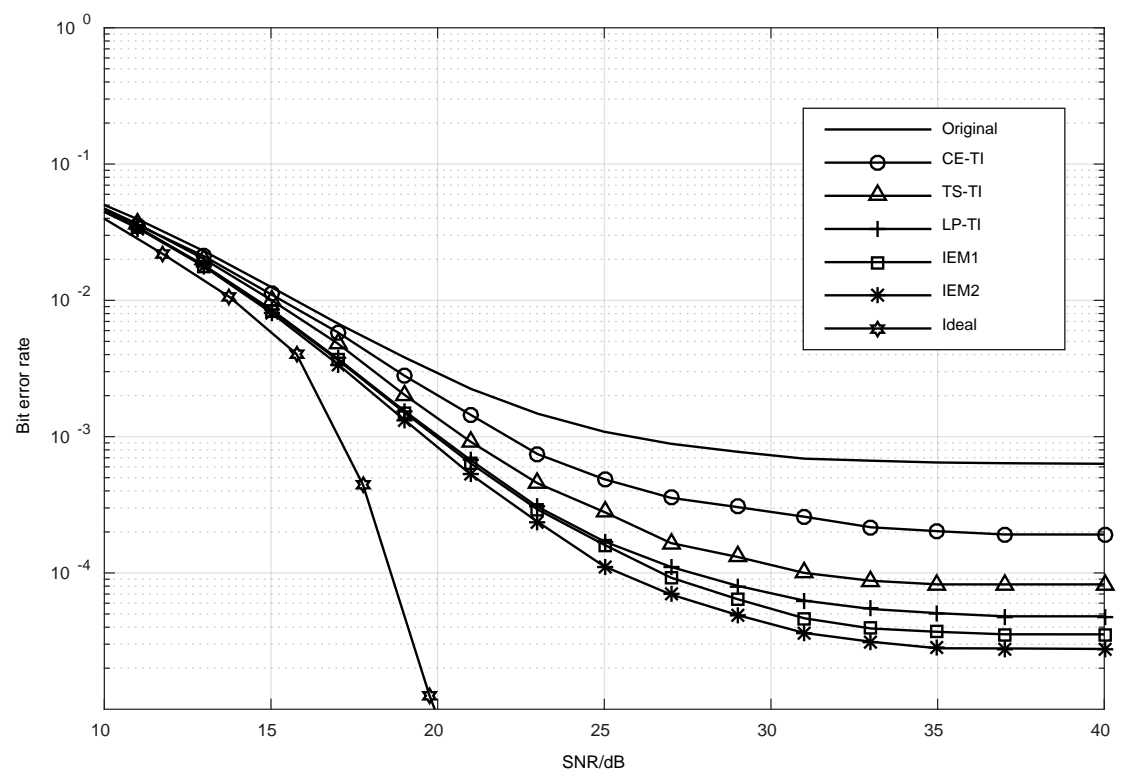

Fig. 8. BER of the various schemes with $6.5 \mathrm{~dB}$ SSPA saturation point, 64QAM and 256 subcarriers.

Finally, Fig. 8 compares the BER performance versus $E_{b} / N_{0}$ of different schemes under hexagonal constellation with solid state power amplifier (SSPA), with comparison to that of the original OFDM scheme. Note that the SSPA model, i.e., Rapp model, can be expressed by [1] 


$$
y(t)=\frac{x(t)}{\left[1+\left(\frac{|x(t)|}{C}\right)^{2 p}\right]^{(1 / 2 p)}}
$$

where the parameter $C, x(t)$ and $\mathrm{y}(t)$ and are the saturating point of the SSPA, input signal and output signal, respectively. Here, the parameter $p$ controls the AM/AM sharpness of the power amplifier. In the simulations, we choose $C=6.5 \mathrm{~dB}$ and $p=3$. The number of samples in all PAPR schemes are set to 200. As seen in Fig. 6, to obtain a $10^{-3}$ BER performance of MCM system, the required $E_{b} / N_{0}$ S of the CE-TI, Parallel TS-TI, LP-TI, IEM1 and IEM2 under SSPA are $22.1 \mathrm{~dB}, 20.8 \mathrm{~dB}, 19.9 \mathrm{~dB}, 19.8 \mathrm{~dB}$ and $19.5 \mathrm{~dB}$, respectively. Since side information is eliminated by the hexagonal constellation, therefore, the proposed scheme is propitious to the application.

\section{Conclusion}

While TI is an effective PAPR reduction technique, its computational complexity rises exponentially with the extra equivalent constellation number. To remedy this, a new immune and evolutionary mechanism-based global strategy is presented. It utilizes the vaccination and selection schedules to remove the duplicated work during the search. Monte Carlo simulation results demonstrate that the new algorithm can obtain a better PAPR improvement and lower computational complexity than other schemes.

\section{Appendix}

In this section, the learning model are described as follows:
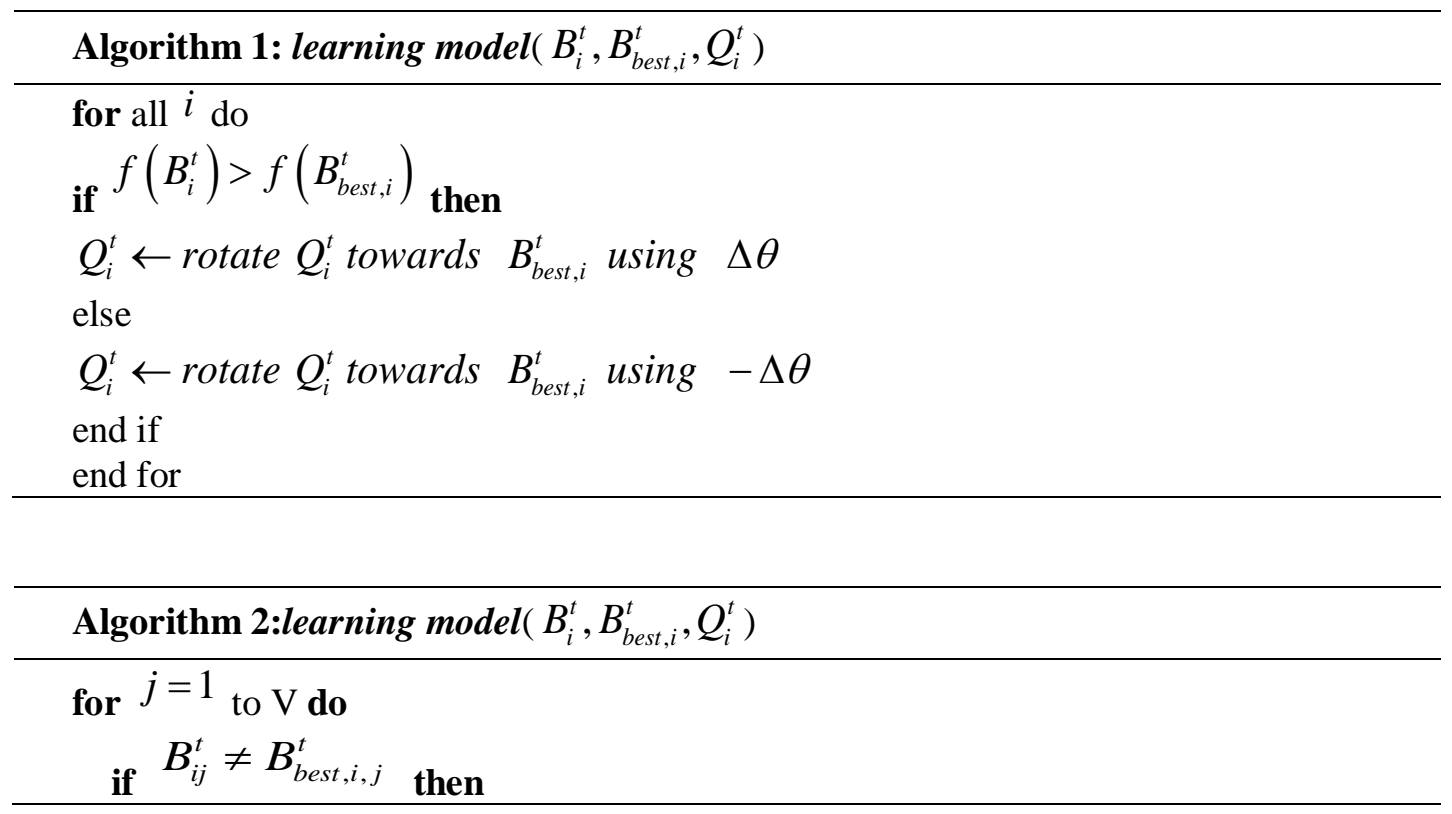


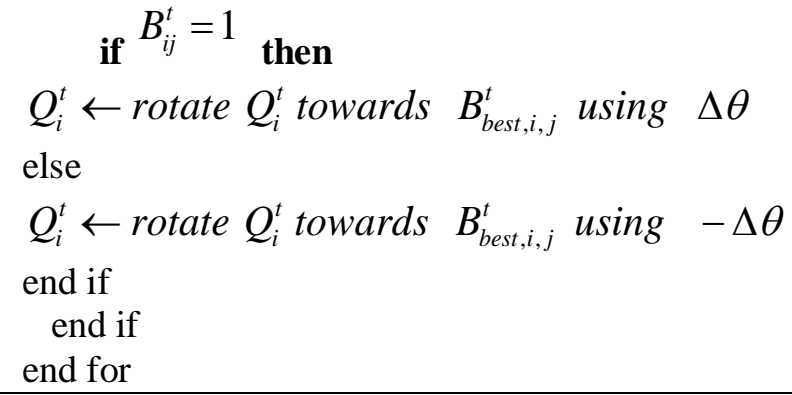

\section{ACKNOWLEDGMENT}

This work was supported in part by the National Natural Science Foundation of China under Grant No. 61501058, the China and Shaanxi Postdoctoral Science Foundation Grant No. 2018M631116.

\section{References}

[1] R. van Nee and R. Prasad, OFDM for Wireless Multimedia Communications, MA: Artech House, Boston, 2000. Article (CrossRef Link)

[2] J. Tellado, Peak to average power reduction for multicarrier modulation, Ph.D. dissertation, Stanford Univ., Stanford, CA, Sep. 1999.Article (CrossRef Link)

[3] J. Tellado, Multicarrier Modulation with Low PAR: Applications to DSL and Wireless, Kluwer Academic Publishers, 2000. Article (CrossRef Link)

[4] S. H. Han, J. H. Lee, "An overview of peak-to-average power ratio reduction techniques for multicarrier transmission,” IEEE Wireless Communications, vol. 12, no. 2, pp. 56-65, Feb. 2005. Article (CrossRef Link)

[5] T. Jiang, and Y. Wu, "An overview: Peak-to-average power ratio reduction techniques for OFDM signals,” IEEE Trans. Broadcast., vol. 54, no. 2, pp. 257-268, Jun. 2008. Article (CrossRef Link)

[6] Y. Rahmatallah and S. Mohan, "Peak-To-Average Power Ratio Reduction in OFDM Systems: A Survey and Taxonomy," IEEE Communication Surveys Tutorials, vol. 15, no. 4, pp. 1567-1592, Nov. 2013. Article (CrossRef Link)

[7] S. Mazahir and S. A. Sheikh, "Optimal Design of Piecewise Linear Companding Transforms for PAPR Reduction in OFDM Systems," KSII Transactions on Internet and Information Systems, vol. 10, no. 1, pp. 200-220, 2016.

[8] A. A. Abdulkafi, M. Y. Alias, Y. S. Hussein, N. Omar and M. K. B. Salleh, "A Hybrid PAPR Reduction Scheme for Optical Wireless OFDM Communication Systems," KSII Transactions on Internet and Information Systems, vol. 12, no. 3, pp. 1136-1151, 2018. Article (CrossRef Link)

[9] S. H. Han, J. M. Cioffi, J. H. Lee, "Tone injection with hexagonal constellation for peak-to-average power ratio reduction in OFDM," IEEE Commun. Lett., vol. 10, no. 9, pp. 646-648, Sep. 2006. Article (CrossRef Link)

[10] M. Ohta, Y. Ueda, and K. Yamashita, "PAPR reduction of OFDM signal by neural networks without side information and its FPGA implementation,” Wiley Online Library, vol. 91, no. 4, pp. 52-60, 2008. Article (CrossRef Link)

[11] C. Tuna and D. L. Jones, "Tone injection with aggressive clipping projection for OFDM PAPR reduction," in Proc. of 2010 IEEE International Conference on Acoustics, Speech and Signal Processing, 2010. Article (CrossRef Link)

[12] J.-C. Chen and C.-K. Wen, "PAPR reduction of OFDM signals using cross-entropy-based tone injection schemes," IEEE Signal Process. Lett., vol. 17, no. 8, pp. 727-730, Aug. 2010.

Article (CrossRef Link) 
[13] J. Hou, C. Tellambura, and J. H. Ge, "Tone injection for PAPR reduction using parallel Tabu search algorithm in OFDM systems,” in Proc. of IEEEGlobecom'2012, Anaheim, CA, United states, Dec. 2012. Article (CrossRef Link)

[14] N. Jacklin, and D. Zhi, "A linear programming based tone injection algorithm for PAPR reduction of OFDM and linearly precoded systems,” IEEE Trans Circuits and Systems Part I, vol. 60, no. 7, pp. 1937-1945, Jul. 2013. Article (CrossRef Link)

[15] J. Hou, X. M. Zhao, F. K. Gong, F. Hui and J. H. Ge, "PAPR and PICR Reduction of OFDM Signals with Clipping Noise-based Tone Injection Scheme,” IEEE Trans. Veh. Technol., vol. 66, no.1, pp. 222-232, Jan. 2017. Article (CrossRef Link)

[16] K. H. Han and J.H. Kim, "Quantum-inspired evolutionary algorithm fora class of combinatorial optimization,” IEEE Trans. Evol. Comput., vol. 6, no. 6, pp. 580-593, Dec. 2002.

Article (CrossRef Link)

[17] J. Hou, J. H. Ge, and S. T. Huang, "Immune evolutionary algorithm to reduce PAPR of OFDM signals using PTS technique,” in Proc. of GLOBECOM 2011, pp. 1-5, Dec. 2011. Article (CrossRef Link)

[18] M. D. Platel, S. Schliebs, and N. Kasabov, "Quantum-inspired evolutionary algorithm: A multimodel EDA,” IEEE Trans. Evol. Comput., vol.13, no. 6, pp. 1218-1232, Dec. 2009. Article (CrossRef Link)

[19] L. C. Jiao, L. Wang, "A novel genetic algorithm based on immunity,” IEEE Trans. Syst., Man, Cybern. A, Syst., Humans, vol. 30, no. 5, pp.552-561, Sep. 2000. Article (CrossRef Link)

[20] C. Tellambura, "Computation of the continuous-time PAR of an OFDM signal with BPSK subcarriers,” IEEE Commun. Lett., vol. 5, no. 5, pp.185-187, May 2001. Article (CrossRef Link)

[21] A. Pezeshk and B. H. Khalaj, "Extended hexagonal constellations as a means of multicarrier PAPR reduction,” in Proc. of EurAsia-ICT'2002, vol. 2510, pp. 926-936, 2002. Article (CrossRef Link)

[22] 3GPP TSG RAN WG1, Motorola TDoc R1-040522, "Comparison of PAR and cubic metric for power de-rating," May 2004.

[23] 3GPP TSG RAN WG1, Motorola TDoc R1-060023, “Cubic metric in3GPP-LTE,” Jan. 2006.

[24] A. Rabson, I. M. Roitt, P. J. Delves, Really Essential Medical Immunology, Wiley-Blackwell Press, 2004. Article (CrossRef Link)

[25] K. Lee, M. E. Sharkawi, Modern Heuristic Optimization Techniques: Theory and Applications to Power Systems, Wiley-IEEE Press, 2008. Article (CrossRef Link) 


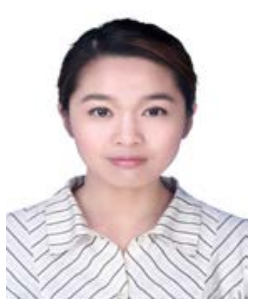

Yang Zhang received the B.S. and the M.S. degrees in communication and information system from Xidian University, Xi'an, Shaanxi, China in 2011 and 2014, respectively. She is currently a Ph.D student with the school of Information Engineering, Chang'an University, Xi'an, Shaanxi, China. Her research interests include wireless communication and signal processing in intelligent transportation systems.

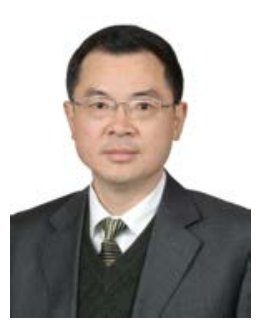

Xiangmo Zhao received the Ph.D. degrees from Chongqing University, Chongqing, China in 1989. Dr. Zhao is currently a full Professor and Vice president of the Chang'an University, Xi'an, Shaanxi, China. He has authored or co-authored over 130 publications and received many technical awards for his contribution to the research and development of intelligent transportation systems. His research interests include intelligent transportation systems, distributed computer network, wireless communication and its signal processing.

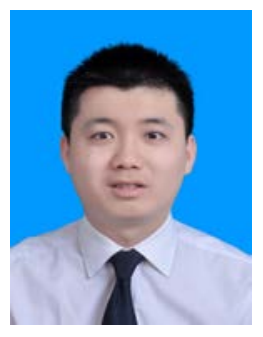

Jun Hou received the B.S. and the Ph.D. degrees in communication and information system from Xidian University, Xi'an, Shaanxi, China in 2007 and 2013, respectively. From 2012 to 2013, he was a joint student in the Department of Electrical and Computer Engineering at the University of Alberta, Edmonton, AB, Canada. Dr. Hou is currently an Assistant Professor with the school of Information Engineering, Chang'an University, Xi'an, Shaanxi, China. His research interests include wireless communication and its signal processing, intelligent transportation systems, and internet of things.

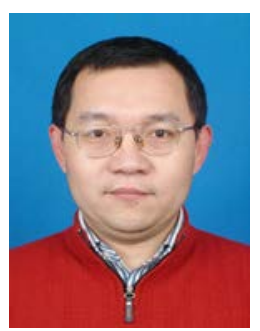

Yisheng AN received the M.S. and Ph.D. degree from Xi'an Jiaotong University, Xi'an, Shaanxi, China in 2010. Dr. An is currently a full Professor with the school of Information Engineering, Chang'an University, Xi'an, Shaanxi, China. He has authored over 40 publications and received many technical awards for his contributiont of intelligent transportation systems. His research interests include distributed system modeling and simulation, traffic signal control and optimization, and intelligent transportation system. 